INTERNATIONAL DESIGN CONFERENCE - DESIGN 2018

https://doi.org/10.21278/idc.2018.0504

\title{
ANALYSIS OF CO-DESIGN SCENARIOS AND ACTIVITIES FOR THE DEVELOPMENT OF A SPATIAL-AUGMENTED REALITY DESIGN PLATFORM
}

\author{
F. Morosi, I. Carli, G. Caruso, G. Cascini, V. Dhokia and F. Ben Guefrache
}

\begin{abstract}
This paper discusses how Spatial Augmented Reality (SAR) can support design sessions in the fields of product, interface and packaging design. We analyse how the scope of a design session and the type of collaboration require different features of the SAR technology. We benchmark a SAR platform under development within the SPARK project (http://spark-project.net/) and state of the art solutions against the proposed classification framework to evaluate the current state of the platform, its limitations and to outline SAR technology requirements for future development possibilities.
\end{abstract}

Keywords: spatial augmented reality (SAR), mixed prototype, collaborative design, participatory design, design creativity

\section{Introduction}

Idea generation is the first essential process in all industrial design activities: given a problem or defined a set of requirements, the designers need to create a group of suitable possible solutions to address them. Despite its importance, only a few ICT tools exist to support the idea generation phase. In most cases, they replicate in digital form the tools typically used to facilitate brainstorming sessions (e.g. post-it notes, whiteboards, mind maps etc.) or they provide access to relevant external knowledge (e.g. patents in other fields of application addressing similar problems). However, there are no solutions on the market specifically tailored to enhance collaborative idea generation activities involving design practitioners and other people (e.g. customers, end-users) with limited or no expertise in design. This scenario, often referred to as participatory design, is becoming more common especially in those fields where the feelings of the customers is extremely important and difficult to measure and ascertain. For instance, the design of product interface or packaging.

Among the challenges faced in participatory design activities, language barriers - the difficulty of nondesigners to visualize what designers propose and to express own desires and thoughts - constitute a significant limitation and can hinder the fruitful exchange of information between designers and other stakeholders.

ICT technologies might play a relevant role in enabling a smoother interaction in participatory design activities, e.g. allowing the visualization of rough or early concepts in a realistic way that does not require technical skill to make an appropriate interpretation of what other participants are saying. Mixed Reality (MR) solutions where digital and physical content co-exist and interact in real time in a physical or virtual world, are in principle good candidates to play this role. Having the possibility to test these technologies in real use cases could help to benchmark them against standard approaches and define the basis of new user interface paradigms based on different interaction metaphors. Nevertheless, high 
equipment cost, high expertise level required and high dependence on the specific application have so far limited the successful exploitation of MR technologies in industry. Among the existing technologies, Spatial Augmented Reality (SAR) can support idea generation activities by combining physical and digital prototypes, i.e. the rough shape and the external finishing respectively, and by providing a more natural manipulation of the mixed mock-up, i.e. a direct interaction for the end users with the real model and a simplified Human-Computer Interface (HCI) for the management of the virtual augmented contents. This is a technological variation of the Augmented Reality (AR) paradigm which overcomes the technical and ergonomic drawback of MR thanks to the use of a spatial display (Bimber and Raskar, 2005). The innovative aspect of this technology is the shared view of the mixed prototype which the design session is working on. Each participant observes in real time and with respect to his/her point of view, the results, and can interact directly with the real model and make comparisons between different versions. Displaying information in a 3D space ensures a more immersive visualisation, a more direct manipulation, an easier navigation and a better comprehension with respect to pure digital prototypes. These the predominant reasons why SAR/AR technologies are investigated within industrial sectors that require a better understanding of complex spatial arrangements (Seichter, 2003).

In this paper, we present some initial results of the SPARK project (SPatial Augmented Reality as a Key for co-creativity - http://spark-project.net/), an innovative SAR environment made for supporting the collaborative design activities that typically take place in design agencies. The main objective of this tool is to reduce the language barriers among the members of a multidisciplinary team by providing an engaging, simple to use and as participative as possible interaction with the prototype. The technology allows the fast arrangement of the outer layout of the product, as a consequence of the real-time visualisation of the modifications, and can be adopted at the early stages of the development. These factors can drastically reduce the number of iterations that usually take place during the entire design process (from the idea generation phase to the final production) and, at the same time, they can enhance the creativity of the actors involved in the collaborative sessions.

The aim of this paper is to analyse our SAR platform from the user perspective by considering two relevant aspects that influence the technology involved: the scope of the design session (i.e. the activity) and the type of collaboration (i.e. the scenario). These, as well as the design fields target of our research, are described in detail in Section 2. Examples of state-of-the-art technologies are discussed in Section 3 , taking into consideration the proposed classification of design scenarios and design activities. The Section 4 provides some implementation details of the SPARK system focusing on the session room setup, the design session preparation, the interaction with the mixed prototype and their impact on the overall functioning of the platform. Section 5 positions SPARK with respect to the same classification proposed in Section 2. Based on the results of this discussion and on some specific tests carried out in a controlled environment with the involvement of professional and non-expert users, possible developments of SAR systems for co-design are outlined in Section 6.

\section{Design scenarios and design activities}

Design scenarios can be classified according to the participation of single or groups of individuals. Maher et al. (2011) make a classification of Individual, Collaborative and Collective design spaces that are taken as reference in this research to describe the characteristics of the design activities that occurs in each category. Nevertheless, the third category here is defined as participatory because of the smaller scale of design activities in respect to the large scale of the collective ones. The three categories make use of virtual and physical design representations, as proposed in the work of Pei et al. (2009) to share and effectively communicate design intentions.

\subsection{Design scenarios}

We refer to individual design when a single, typically expert designer runs the ideas generation and prototyping activities on their own. The individual designer generates new design ideas autonomously and has periodic meetings with the stakeholders involved in the project to review, obtain feedback and assess the advancements of the work. Normally, the designer adopts the design tools with which they feel most comfortable with and follow this approach to generate design ideas. 
Collaborative design activities are run by a team of design practitioners possibly with different levels of expertise, diverse technical communication skills and alternative approaches to design. In the implementation of design activities, multiple concepts and ideas are discussed and shared with the team in order to receive feedback and determine the next cycle of iterations before presenting to the client the different design proposals. The level of abstraction of the design representations can be higher than the one in the scenario with non-expert participants. Design practitioners in a collaborative session can all actively modify the design representations. Participatory design involves co-creative sessions with different stakeholders that typically do not share a univocal perception of the product, nor the same communication language. The aim of these activities is to reduce the number of alternative concepts to be developed further and possibly to get inspiration for improvements. The sessions with the end users and consumers are particularly suitable to receive feedbacks for FMCG (Fast Moving Consumer Goods) packaging and for product interface design. The design of outer features, in product interface design, influences the affordance of the product functionalities perceived by the user, its ergonomics, i.e. the reachability of the features, and aesthetics. The non-expert user's direct involvement in the modification of the design representation is typically limited due to their inexperience and lack of design skill. The level of abstraction of the design representations in this scenario needs to be low in order to facilitate the feedback flow from the participants.

\subsection{Design activities}

Whatever design approach or methodology one follows, three essential activities occur, namely analysis, synthesis and evaluation, as for instance documented by Jones (1984), who referred to these as essential design phases. Analysis consists of elaborating on the design requirements and on the definition of related performance specifications. Synthesis is then related to finding potential solutions for each performance specification and building up design proposals with the least amount of compromise possible. The synthesis activities occur during both, the early stage design phase (ES), where novel ideas are generated, and the design refinement phase (DR), where the designer focus is more in generating new proposals on smaller and delimited design problems. Evaluation examines the accuracy with which alternative design solutions fulfil performance requirements before the final design is selected. In the categorisation of the design activities proposed in this paper, a fourth phase called communication, described by Cross (2008), is considered. This phase describes the final design solution and it represents the consequence of many cyclical iterations between the synthesis and the evaluation phases.

\section{Previous AR studies on design scenarios}

Despite the considerable development in recent years of AR-based systems for industrial application, there are only few cases in literature of such technology as a support tool in design creativity (Nee et al., 2012). In this section these examples have been investigated following the design scenario classification previously provided (Section 2).

Tangible Viewports (Gervais et al., 2016) is an example of an SAR application that aims to support a single user through tangible interactions with augmented prototypes and desktop-based interfaces of traditional software. The objective in their work is to provide a more engaging experience in design synthesis activities with a focus on packaging design by taking advantage of the precision of standard interaction paradigms coupled with the intuitive experience based on direct manipulation. The presence of a monitor, in front of which is placed the physical object to create the illusion that is part of the same system, makes this system usable only for an individual design scenario. The hardware setup is simplified (single projector, compact tracking system and commercial gesture recognition device) due to the desktop setup and limited interaction area. More related to product design evaluation activities is the AR application developed by Park (2008), where a single user can test the usability of interfaces interacting directly with the prototype. The efficiency of this type of interaction is possible due to the use of a skin region overlay algorithm that eliminates the superimposition of the augmented images from the user's fingers, a marker-based tracking system and an edge detection function. By giving to the user the possibility to change some of the mock-up's characteristics (shapes, colours, textures), among a set of predefined alternatives, Park extended his tool to enable realistic evaluation activities, where the effectiveness of each configuration can be easily assessed. 
By increasing the number of participant in a design session, the AR/SAR technology is faced with the challenges related to the distribution and the scope of the activity. In this context it is worth mentioning the lab experiment conducted by Ben Rajeb and Leclercq (2013) with the Collaborative Digital Studio (CDS) tool, in which annotations and graphic documents are shared in real time and remotely by using the metaphor of traditional meetings. They create four different spatial configurations of the same collaborative environment to investigate which analysis and communication activities are supported: the expert consultation for sharing knowledge and accessing advices; the collaborative meeting for generating new ideas and sharing points of view collectively; the group review for sharing individual ideas, comments and solutions among the team members; the public evaluation for presenting and evaluating new ideas. Despite its general adaptability, the CDS is not suitable for the idea generation stage, which requires more specific features related to the task.

Applications in synthesis activities of collaborative scenarios are presented in (Shen et al., 2010) where an AR environment, based on Head Mounted Displays (HMD) is used to support product design sessions among members of a multidisciplinary team of experts. In this case the user can observe the prototype from different angles and perspectives to notice and evaluate in real time all the applied modifications. Great emphasis has been placed on the development of a client/server framework for distributed design teams and an intuitive interface in virtual and physical spaces. This latter characteristic was also underlined in the SAR work space developed for architectural design by Calixte and Leclercq (2017), where all the users participating in the communication activity are brought together in the same projection space around the same augmented artefact. They stressed the necessity to improve the interaction metaphors of the interface to simplify the use and interpretations of the tool and consequently make all the designers active during the collaborative session.

Another aspect observed with the state-of-the-art analysis is the possibility to change the session environment. ARUDesigner (Wang and Chen, 2009) is a framework that helps designers estimate the effect of alternative proposals and choose the most suitable. It incorporates AR/VR dual viewing modes (virtual objects combined with a real environment / digital contents inserted in a virtual background) that the users, depending on the necessity, can change at any time during the session: focus on collaboration for the first setup and focus on the immersivity of the system for the second.

The extension of the design activity to a more participatory scenario, where non-expert users can collaborate, has a minimum influence on the type of technology involved, but has a relevant impact on how it works. The Virtual Round Table (Broll et al., 2000) is a clear example of how an AR environment for urban and architectural design analysis should be adapted in order to display onto the augmented prototype abstract metadata, such as stresses and loadings, comprehensible by all the participants. Moving towards more interactive systems for design synthesis activities, attention is growing on the interfaces design, as well as the definition of the interaction metaphors with the augmented prototypes and the other participants. SeamlessDesign (Kiyokawa et al., 1999) and MxR (Belcher and Johnson, 2008) are both virtual/augmented environments where users, by wearing HDM, can modify digital and/or physical contents starting from the early stages of development. In the first application, the use of avatars within the blind (i.e. fully virtual) mode has preserved face-to-face collaboration; inversely, in the second the availability of specific physical tools has increased the interaction possibilities. With this latter solution, in particular, all the participants managed to perform relatively complex tasks with a small set of instructions and the time needed to learn the tangible interface seems to be comparable with a desktop-based GUI.

Within the participatory scenarios, the literature has investigated how the geographical and temporal distributions of people involved in collaborative session impact on the overall architecture of the SAR/AR platform. This becomes relevant in the work done by Chryssolouris et al. (2009) where dispersed actors can review and slightly customise the outputs of the product design activity at their own premises. The web-based framework of the system integrates and synchronizes the interfaces with the virtual/augmented reality visualisations and stores all the product-related data in a database for managing the product versions after the end of the session. Also the system developed by the European Computer-Industry Research Center (Ahlers et al., 1995) deals with the geographically remote collaboration by means of web-connected workstations. This latter work, in particular, is focused more on the communication design activity where a potential customer has the possibility to decorate their 
own room by selecting furnitures from a predefined catalogue. The innovative aspect is the introduction of the "Facile" language, which allows non-experts to correctly setup a shared AR application thanks to the use of separated channels for the functional paradigms.

\section{SPARK system implementation}

To set up a collaborative design session supported by the SAR technology, where several users can interact around a mixed prototype that dynamically changes as the design activity evolves, multiple hardware and software components need to work together seamlessly. The current SPARK setup has been implemented in a lab environment and is comprised of two projectors as the visualization system, the physical blank prototype (mock-up), the mock-up tracking system, the user interaction device (tablet) and the web interface (IS Server) for the preparation of the design assets (graphic elements and 3D models) and for remote collaborative attendance. These components form the platform architecture (Figure 1) and are described in detail in the following sub-sections. The components, are adaptable according to the needs and constraints of the target design agency.

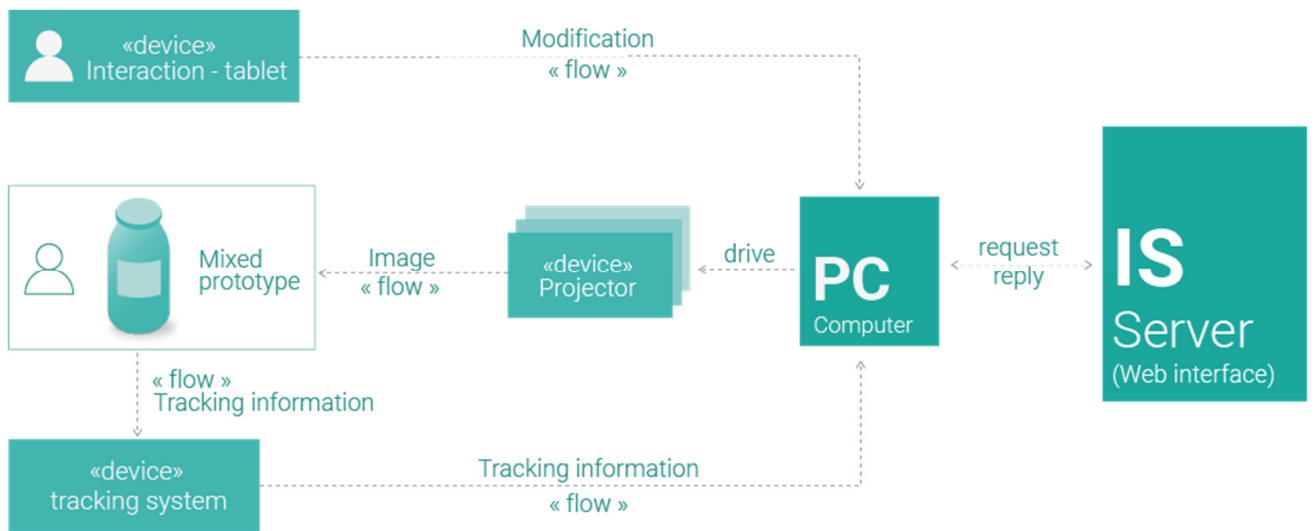

Figure 1. SPARK platform architecture: Main components and information flows

\subsection{Setup of the session room}

Projectors and tracking system are hardware components that need to be calibrated each time the layout of session room is changed. The graphical elements applied to the mixed prototype are displayed onto the blank mock-up by means of the projected images. The adaptability of the current setup allows for the number of projectors to be changed according to the desired projection volume and the dimension of the real prototype. The projectors are directly connected to a graphic engine which reconstructs the system (projectors and mock-up) in a digital 3D environment. The software also manages in real time what images should be projected and how the multi-projection should be overlapped.

The recording of the real-time position of the mock-up is provided by an array of optical devices (infrared cameras and infrared light emitters), connected with each other and driven by a computer. The setup of these devices is relevant for defining the tracking volume, i.e. the working space where the user can manipulate the prototype, and the available degree of freedom for the manipulation itself, i.e. the limitation for the rotational movements. In the current setup, the tracking workspace covers most of the table surface, but is limited by the projection area. The mock-up should be equipped with infrared reflective markers positioned so that are clearly visible from all the cameras and without any repetition or symmetry. The dimension of these markers can vary, a smaller dimension is usually preferable if the only available surface is also used to display design representations during the collaborative session activities (they may cause a performance reduction).

\subsection{Session mock-up and assets preparation}

The blank mock-ups are produced by means of traditional or rapid prototyping techniques, also existing white painted products or packaging can be used. The mock-up surface should not be excessively rough or shiny and is crucial to obtaining a good quality visualization of the mixed prototype. 
The design assets should be prepared by the designer before the beginning of the session. The 3D models, that represent the exact digital versions of the mock-up, consist of polygonal meshes at different stages of development, i.e. 3D models at the early stage and design refinement. The graphic elements consist of single images that the designers generate by means of commonly used software image editors such as Adobe Illustrator and Photoshop. The 3D models and graphic elements, after their creation, are ready to be uploaded on the web interface (IS server) where design projects are arranged as clients, products and design sessions. Each session contains the design assets. The web 3D editor enables the designer to build preliminary versions of design representations that can be retrieved at the beginning of the SAR session. While the design session is running, each applied modification is automatically saved and when the session comes to an end, the generated ideas can be reviewed and further explored. The web interface enables remote users to follow in real time all the modifications performed during the design session.

\subsection{Interaction with the mixed prototype}

Two main interactions with the mixed prototype are performed by the session users. The first one concern direct manipulation of the mixed prototype, in which the participants of the session can freely explore the augmented contents by moving and rotating (Figure 3). The second one affects the interaction with the graphic elements projected on the mock-up and the 3D digital model through a tablet device (Figure 2). These tasks are normally performed by the designer, s/he can preview and select a graphic element from the assets library, place it on the 3D model surface, modify its position, layer depth, rotation, scale and change the colour of each part of the 3D model. The tablet screen allows two types of visualization and interaction setup (Figure 2), the first consists in the visualization of the 3D model where the user can place and modify the graphic elements attributes, the second one displays a touch area without displaying the 3D model. The tablet in this mode is working as a touchpad and the mixed prototype as the display. In either setting, the modifications are displayed in real time on the mixed prototype and are performed by means of multi-touch gestures. The user drags a single finger on the touchscreen to translate the graphic element on the prototype surface, the user pinches two fingers inward and outward to scale it and turns two fingers to rotate it clockwise and anticlockwise. The purpose to interact with the mixed prototype features by using multi-touch gestures on a touchscreen, is to provide a well-established and robust interaction solution. Therefore, the overall usability of the system is perceived as more important by the session users than the pixel resolution and precise placement on the mixed prototype.
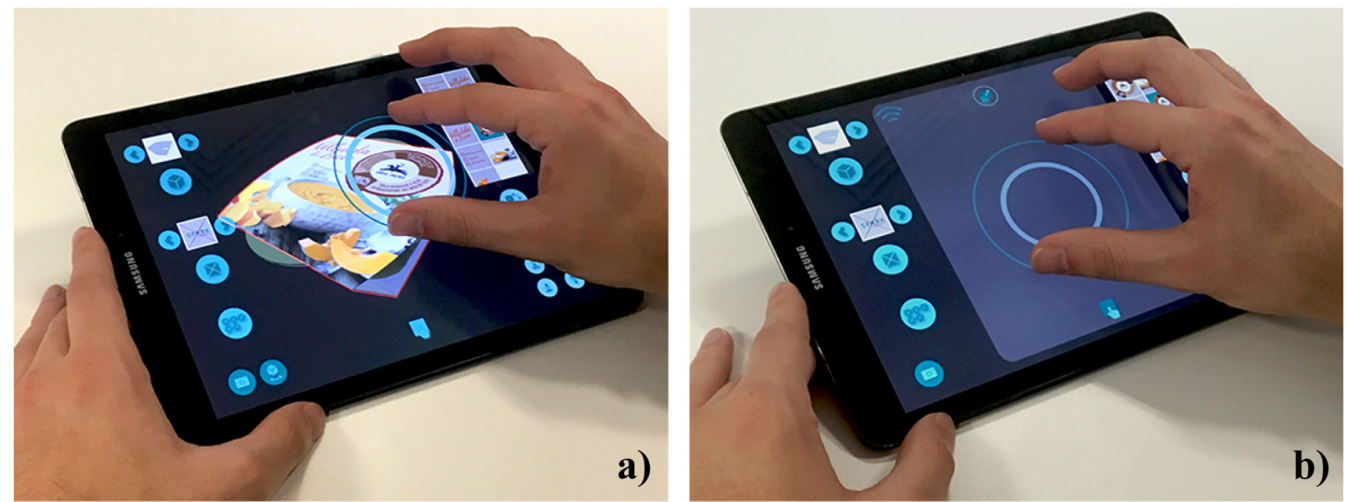

Figure 2. Different visualization modalities: a) 3D model, b) touch area

\section{Design scenarios requirements for SAR technology}

This section analyses the current state of development of the SPARK platform by considering the framework of the design scenarios and activities previously discussed. As a result of the testing carried out in a controlled environment sufficient user feedback was captured to establish the different purpose of utilization and the impact of user profiles on the SAR technologies. The detailed description of how these tests has been made is out of the scope of the current paper (for further details see Becattini et al., 2017), but they have involved both design practitioners and non-expert users. 
Figure 3 illustrates a specific session related to packaging design during the tests: the two projectors and the optical tracking system are mounted on a ceiling structure pointing from opposite directions on a limited side area of the meeting table (the black zone on which is placed the mixed prototype). The participants sit around the remaining part of the desk to reduce the risk of occlusions and can perform all the modification by acting on the tablet interface or by asking the designer in charge of using it.

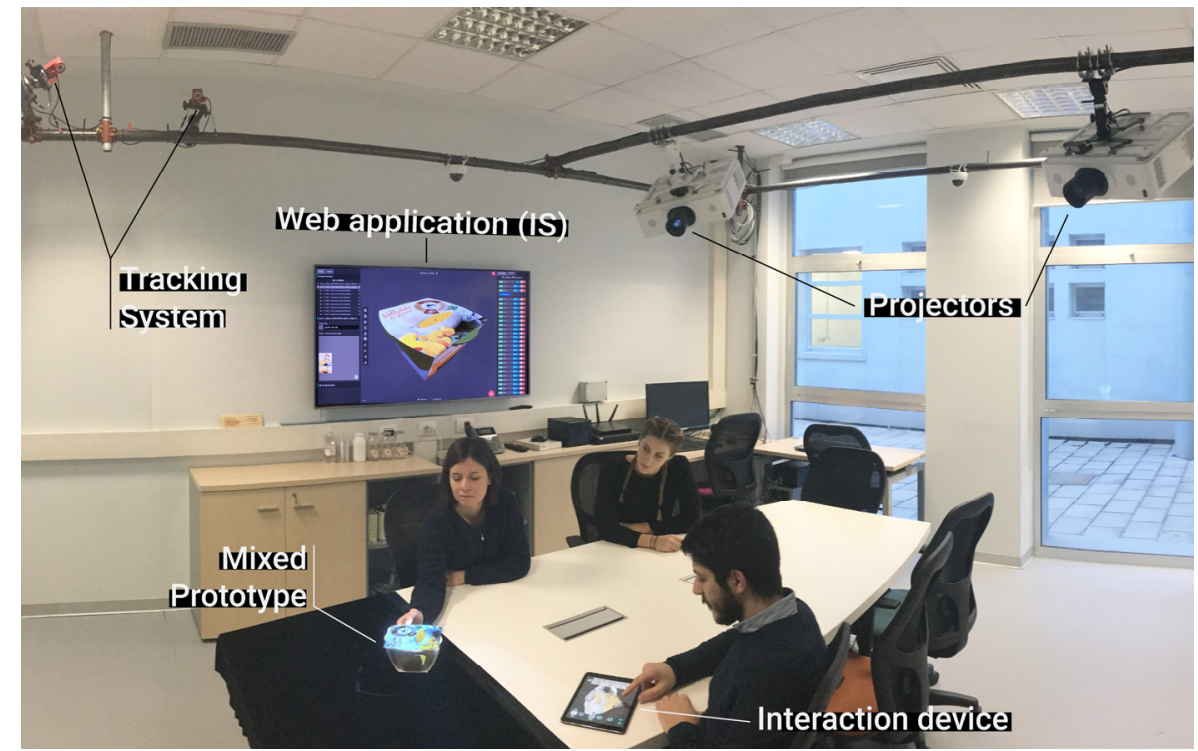

Figure 3. SPARK platform's main components at work during a collaborative session

\subsection{Individual design}

With an individual design scenario, the SAR system can be decreased to a more compact and simplified setup. Since there is only one designer, a traditional work desk space is adequate to provide a wide enough point of view on the mixed prototype and a sufficient interaction area. A single projection system can be used and installed in the optimal position accordingly with the user working location: this allows to control with more flexibility the occlusion-related problems. The reduced projection distance is also relevant for the definition of the projector attributes in terms of technical features and price. Further, complex motion capture systems are not justified within this scenario and they can be substituted by compact and low-cost devices or different tracking algorithms. In the latter case, it is possible to ensure the same or even better quality of visualization despite limited mock-up manipulation (i.e. translations and rotations jointed with a tracked planar surface) relevant during the evaluation and the design refinement phases of the synthesis activities.

The individual scenario has a huge impact also for the definition of the interaction with the prototype's augmented content: there is no need for a portable touch screen surface since the mixed prototype can be placed close to the user. Therefore, a traditional input system on a 2D monitor can be used in order to provide a precise and well-established interaction metaphor with the Graphic User Interface (GUI) and the displayed design assets.

The desktop configuration allows a simplification of the system architecture since all the components are driven directly by the same computer. This has relevant impact especially on the hardware's calibration procedure and the management of the session through the web interface. In addition, while some of the features provided by the IS (i.e. the remote visualization and the session preparation) are less relevant within this scenario, there are others (i.e. the review of modifications) that are still necessary to improve the effectiveness of the system and justify its adoption even for individual uses.

\subsection{Collaborative design}

With multiple design practitioners working together on the same SAR environment, a shared setup is required. In addition, thanks to the trained abstraction capability of the users and their knowledge, it is 
possible to assume that the current limitations of the system do not excessively affect the impact of the technology within the design activities; this is especially true during the early stages of development, where designers have more opportunity to experiment with different ideas.

In this scenario, the multi-projection system is relevant to cover most of the mock-up's surfaces in order to provide to the participants simultaneous and inclination-independent points of view of the mixed prototype. Since all the projectors are directed towards the same area with a complete overlap of the images, the projection volume results limit and do not allow every user to comfortably reach and manipulate the mixed prototype. The adopted configuration provides a manipulation volume much smaller than the tracking volume, approximately $400 \times 400 \times 400 \mathrm{~mm}$, and it is located on a side of the meeting table to avoid occlusions with the projected images and the reflective infrared markers.

The collaboration activities during the sessions should be supported with also the interface. In the actual setup only one user at a time can apply modifications on the design representations but, thanks to the portability of the device, it can be easily exchanged without reducing the mobility of the attendees. For what concerns the usability of the interface we provide a set of simplified functions to support an agile navigation with the GUI even without initial training. This can be considered an advantage during the analysis and the early synthesis activities, where fast changes are required, but it is less appropriated for the remaining ones where designers seek high precision and advanced features.

The presence of the IS supports better the user's needs of the collaborative scenario, in comparison with an individual use, thanks to the importance of the synchronous and asynchronous cooperation. With the client/server framework, in fact, it is possible to have remote designers capable of seeing in real time all the modifications applied on the mixed prototype even from different locations. In addition, since the server records all the steps performed during the sessions, the collaborative activities can be staggered without losing any progress. This last functionality is also relevant for the evaluation and communication design activities where the evolution of the product is often reviewed.

\subsection{Participatory design}

A group of practitioners working together with clients and end users in a participatory design session, requires a flawless experience with the SAR environment, a flexible setup and a highly engaging, easy to use user interface. The perceived robustness of the platform is extremely important in this scenario so as not to limit the participation of stakeholders because of mistrust in manipulating the mixed prototype or interacting with the interface.

The multi-projection setup should be redefined: the side volume, where it is allowed the manipulation of the mock-up, is often seen by non-expert users as disconnected from the shared space (i.e. the cowork space). For this reason, with a participatory design, the projection volume has to be increased and shifted in the middle of the meeting table by changing the number/disposition of projectors and by reducing the overlay of the projected images. The first one ensures an expansion of the wrapping region on the mixed prototype, and consequently the number of simultaneous point of views, while the second provide higher visualisation quality due to a reduced use of blending techniques. As for the collaborative, even in this scenario the improvements for the projection and tracking systems are mostly recommended during the synthesis and evaluation activities.

The interaction with the graphic features on the mixed prototype should be engaging and be extensively shared among the participant. Non-expert users are expected to practically contribute to the design activities and, for this, they should be enabled to interact directly with the virtual/digital contents in order to propose their own modifications and design ideas. This possibility is not supported enough with the current setup and can be considered a strong limitation for the creativity of introverted non-expert users.

\section{Possible developments of SAR technologies for co-design}

Thanks to the experience gained in the experimental activities of the SPARK project, we propose in this section an overview of possible developments of SAR technologies for co-design activities. Most of these features are going to be embedded in a next release of the SPARK platform and are inspired by the analysis of previous works in the field. In Table 1 we relate the current and the future developments of SPARK with the literature analysed in Section 3; these are also distinguished according to the SAR (S) and AR (A) environments in which they have been implemented. 
Table 1. Technological features already developed in previous works

\begin{tabular}{|c|c|c|c|c|c|c|c|c|c|c|c|c|c|c|c|c|c|c|c|c|}
\hline \multirow{3}{*}{$\begin{array}{l}\text { Literature } \\
\text { Park (2008) } \\
\end{array}$} & \multirow{2}{*}{\multicolumn{7}{|c|}{ Current state }} & \multicolumn{13}{|c|}{ Possible developments } \\
\hline & & & & & & & & \multirow{2}{*}{ A } & \multirow{2}{*}{$\mathrm{B}$} & \multirow{2}{*}{$\mathrm{C}$} & \multirow[t]{2}{*}{$\mathrm{D}$} & \multirow{2}{*}{ E } & \multirow[t]{2}{*}{$\mathrm{F}$} & \multirow[t]{2}{*}{$\mathrm{G}$} & \multirow[t]{2}{*}{$\mathrm{H}$} & \multirow{2}{*}{$\begin{array}{l}\mathrm{I} \\
\mathrm{A}\end{array}$} & \multirow[t]{2}{*}{$\mathrm{J}$} & \multirow[t]{2}{*}{$\mathrm{K}$} & \multirow[t]{2}{*}{$\mathrm{L}$} & \multirow[t]{2}{*}{$\mathrm{M}$} \\
\hline & & & & $\mathrm{A}$ & & & & & & & & & & & & & & & & \\
\hline Gervais et al. (2016) & & & & $\mathrm{S}$ & & $\mathrm{S}$ & & & & $\mathrm{S}$ & $\mathrm{S}$ & & & & & & & & & \\
\hline $\begin{array}{l}\text { Ben Rajeb and Leclercq } \\
(2013)\end{array}$ & & & & & $\mathrm{S}$ & & $\mathrm{S}$ & & $\mathrm{S}$ & & & & & & & & & & $\mathrm{S}$ & \\
\hline Shen et al. (2010) & & & & & & $A$ & A & & & & $\mathrm{A}$ & & & $\mathrm{A}$ & & & & $\mathrm{A}$ & & \\
\hline Wang and Chen (2009) & & & & & A & A & & & & & & & & A & & & & & & \\
\hline Calixte and Leclercq (2017) & $\mathrm{S}$ & $\mathrm{S}$ & & & & & & & & & & & & $\mathrm{S}$ & & & & $\mathrm{S}$ & $\mathrm{S}$ & \\
\hline Broll et al. (2000) & & & & & & $A$ & & & & & & & & $\mathrm{~A}$ & & & & & & \\
\hline Belcher and Johnson (2008) & & & & & & & & & $\mathrm{A}$ & & & & & & $A$ & & & & & A \\
\hline Kiyokawa et al. (1999) & & & & & & A & & & & & & & & $\mathrm{A}$ & $\mathrm{A}$ & $\mathrm{A}$ & & & & A \\
\hline Chryssolouris et al. (2009) & & & & & & & $\mathrm{A}$ & & & & & & & & & & & & $\mathrm{A}$ & A \\
\hline Ahlers et al. (1995) & & & & & & & $\mathrm{A}$ & & & & & & & $\mathrm{A}$ & & & & & & \\
\hline & ấ. & & $\begin{array}{l}0 \\
0 \\
0 \\
0\end{array}$ & 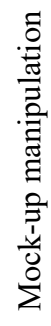 & 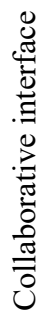 & 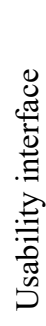 & 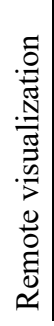 & 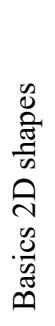 & 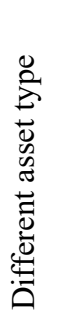 & $\begin{array}{l}\vec{Z} \\
0 \\
\Xi \\
\Xi \\
0 \\
0 \\
0 \\
0 \\
0 \\
0\end{array}$ & 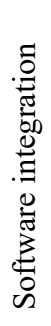 & 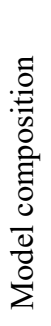 & $\begin{array}{l}\text { ]્ల } \\
\frac{0}{0} \\
\frac{0}{0} \\
\sum\end{array}$ & 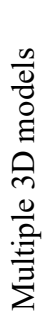 & 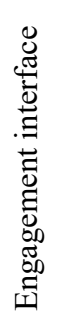 & 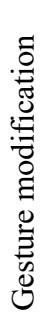 & 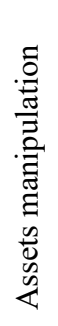 & 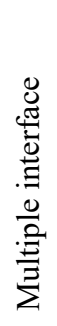 & 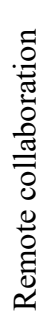 & 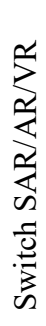 \\
\hline
\end{tabular}

The innovative features are here below classified in 5 major areas (i.e. the aspects to improve): creativity, engagement, collaboration, abstraction and visualisation.

\section{Creativity (2D design assets):}

- $[\mathrm{A}]$ the assets library should contain digital $2 D$ shapes (i.e. square, circle, triangle, etc.) that can be used as placeholder for assets not available in the library and to create original compositions. This functionality, adapt for packaging and interface design during the early stages, is not suitable for participatory scenarios because non-expert users do not have the same abstraction capacity as the designer or the same ability to complete more complex modification tasks.

- [B] the assets library should accept different types of design assets (i.e. textures, animated GIF, fonts, etc.) that are important especially for the rapid-prototype of interfaces. These types of components are usually discussed during refinement sessions and, by providing only a finite set of customisation possibilities that can be easily operated by non-experts.

- [C] the assets library should accept new assets even while the session is going on. During the early stages of development not all the graphical elements are defined. For this reason, having the possibility to browse in other databases (web or local) can really increase the potential impact of the session.

- $[\mathrm{D}]$ the platform should be integrated with professional software commonly used by experts in respective fields. This functionality, which allows designers to easily modify the assets already loaded in the database (as supposed in $[\mathrm{C}]$ ), is relevant for the design refinement phase when even the smallest details are discussed.

\section{Creativity (3D design assets):}

- [E] the physical prototype can be decomposed in to different parts/bricks (all tracked) to be used to build a new version of the mixed prototype. This functionality, relevant for the initial design activities (analysis and synthesis) of product design, implies development of new tracking methods or algorithms (i.e. infrared texture recognition). 
- $[\mathrm{F}]$ the shape of the physical prototype might be easily modified by using clay models. This feature, that support early-stage innovation of product design, should be integrated with 3D scanning techniques in order to obtain the digital mesh to be used inside the platform.

- [G] the platform should be able to manage simultaneously more than one mixed prototype per session. This functionality allows for the comparison between different prototypes or different versions of the same prototype. It is important during the analysis and evaluation activity.

\section{Engagement (user interface/interaction):}

- $[\mathrm{H}]$ the interface could be improved in terms of engagement and intuitiveness in order to increase the participation of non-experts during the design session. This need can be solved by introducing innovative interaction paradigms, like tangible elements or projected surfaces.

- $[\mathrm{I}]$ the interface could be extended also to gesture recognition devices, which allows for applying simultaneous modifications by operating on to the mixed prototype. This feature, that can be considered a consequence of the previous one, is more adapt to participatory sessions.

- $[\mathrm{J}]$ the interface could be improved in terms of editing functionalities. During the design refinement phases, it is important for the designers to have specific functions that increase their precision for the asset's manipulation.

- $[\mathrm{K}]$ the interface could allow multiple and simultaneous interactions with the participants. This functionality, relevant during all the design activities of collaborative and participatory scenarios, can be achieved by increasing the dimension of the interactive surface or by multiplying the number of the interaction devices.

Table 2. Relation between technological features and design activities / scenarios

\begin{tabular}{|c|c|c|c|c|c|c|c|c|c|c|c|c|c|c|c|c|c|c|c|c|c|c|c|c|}
\hline & DesigI & & & & & & & & & & & & & & ssi & ble & $\mathbf{v}$ & op & er & & & & & \\
\hline & Activit & & & & & & & & & A & $\mathrm{B}$ & $\mathrm{C}$ & $\mathrm{D}$ & $\mathrm{E}$ & $\mathrm{F}$ & $\mathrm{G}$ & $\mathrm{H}$ & I & $\mathrm{J}$ & $\mathrm{K}$ & $\mathrm{L}$ & $\mathrm{M}$ & $\mathrm{N}$ & $\mathrm{O}$ \\
\hline $\bar{\sigma}$ & Analys & & $\bullet$ & $\bullet$ & & $\bullet$ & & & & $\bullet$ & $\bullet$ & & & - & $\bullet$ & $\bullet$ & & & & & & & & \\
\hline 를 & & ES & $\bullet$ & $\bullet$ & & $\bullet$ & & & & $\bullet$ & & $\bullet$ & & $\bullet$ & $\bullet$ & & & & & & & & & \\
\hline$\frac{2}{2}$ & Esis & DR & & $\bullet$ & & $\bullet$ & & & & & $\bullet$ & & $\bullet$ & & & & $\bullet$ & & $\bullet$ & & & $\bullet$ & $\bullet$ & \\
\hline 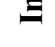 & Evaluat & & & $\bullet$ & & $\bullet$ & & & & & $\bullet$ & & & & & $\bullet$ & & & & & & $\bullet$ & $\bullet$ & \\
\hline 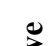 & Analys & & $\bullet$ & & $\bullet$ & $\bullet$ & $\bullet$ & $\bullet$ & $\bullet$ & $\bullet$ & $\bullet$ & & & $\bullet$ & $\bullet$ & - & & & & $\bullet$ & • & & & • \\
\hline • & $\mathrm{S}$ & $\mathrm{ES}$ & $\bullet$ & & $\bullet$ & $\bullet$ & & $\bullet$ & $\bullet$ & $\bullet$ & & - & & $\bullet$ & $\bullet$ & & & & & $\bullet$ & $\bullet$ & & & $\bullet$ \\
\hline 8 & CSIS & DR & & & $\bullet$ & & & & & & 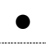 & & - & & & & $\bullet$ & & $\bullet$ & $\bullet$ & $\bullet$ & & $\bullet$ & $\bullet$ \\
\hline$\frac{5}{0}$ & Evaluat & & & & $\bullet$ & & & & & & $\bullet$ & & & & & $\bullet$ & & & & $\bullet$ & $\bullet$ & & $\bullet$ & $\bullet$ \\
\hline & Communic & ion & $\bullet$ & $\bullet$ & $\bullet$ & $\bullet$ & & & & & $\bullet$ & & & & & & & & & & - & $\bullet$ & & \\
\hline P & Analys & & $\bullet$ & & $\bullet$ & $\bullet$ & $\bullet$ & & $\bullet$ & & 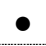 & & & $\bullet$ & $\bullet$ & $\bullet$ & $\bullet$ & $\bullet$ & & $\bullet$ & $\bullet$ & & & $\bullet$ \\
\hline e를 & , & $\mathrm{ES}$ & $\bullet$ & & $\bullet$ & & & & $\bullet$ & & & $\bullet$ & & $\bullet$ & $\bullet$ & & $\bullet$ & $\bullet$ & & $\bullet$ & $\bullet$ & & & • \\
\hline 륭 & & DR & & & $\bullet$ & & & & & & 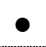 & & $\bullet$ & & & & • & $\bullet$ & & $\bullet$ & $\bullet$ & & $\bullet$ & $\bullet$ \\
\hline 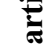 & Evaluati & & & & 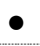 & & & & & & • & & & & & $\bullet$ & & $\bullet$ & & $\bullet$ & $\bullet$ & $\bullet$ & $\bullet$ & $\bullet$ \\
\hline & Communic & ion & & $\bullet$ & $\bullet$ & $\bullet$ & & & & & $\bullet$ & & & & & & & & & & $\bullet$ & ? & $\bullet$ & \\
\hline & & & 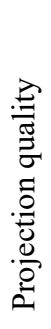 & 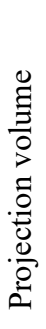 & 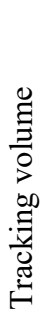 & 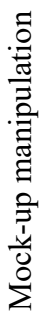 & 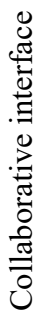 & 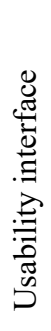 & 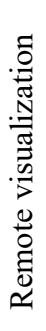 & 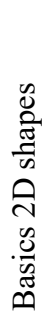 & 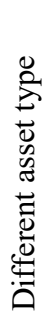 & 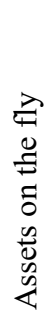 & 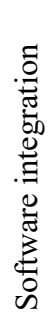 & 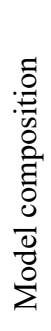 & $\begin{array}{l}\bar{J} \\
\tilde{J} \\
\frac{\infty}{0} \\
\frac{0}{0}\end{array}$ & 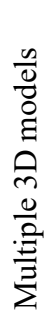 & 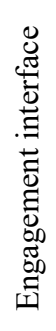 & 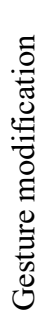 & 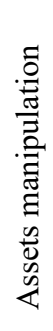 & 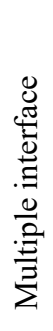 & 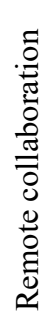 & 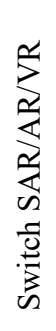 & 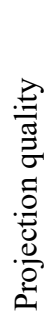 & 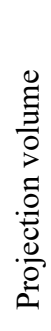 \\
\hline
\end{tabular}

\section{Collaboration (distribution scenarios):}

- [L] The interface and the hardware of the SAR could be duplicated to enable remote collaboration. Both require efficient two-way communication channel between the IS and the 
SAR system. The first one could be used as a secondary means of interaction during the session and the second one implies the definition of low-cost extended versions of the platform.

\section{Abstraction (SAR environment):}

- $[\mathrm{M}]$ the visualization should be changed from SAR to AR/VR environments when it is necessary to overcome the limitations of the mock-up 3D surface in terms of quality. This engaging functionality can provide to the user additional details of the product (i.e. the environment) during the refinement phases of the design activity and reduce the collaboration need.

Visualization (projection quality):

- $[\mathrm{N}]$ the projection should be enhanced in terms of quality and alignment between digital and physical contents. This can be achieved by defining a closer and more perpendicular position of the projectors with respect to the surface of the mock-up, by using high-performance projectors and by improving the results of the calibrations.

- $[\mathrm{O}]$ the projection volume, and therefore the SAR workspace, should be increased without affecting the visual results of the system. This can be achieved by increasing the number of projectors, by reducing their zoom or by increasing their field of view.

In Table 2 we provide a summary description of which features, available at the current state of the platform, can sufficiently satisfy the design activity's requirements for each scenario. We perform the same type of analysis also with the possible future innovations of SPARK: in this case, the table highlights in which scenario/activity the required functionalities have a major impact.

\section{Conclusions}

This paper presented the first implementation of a novel MR system, SPARK platform, developed to support design sessions in the fields of product interface and packaging design. We proposed a classification framework based on the target design scenarios (individual, collaborative and participatory) and activities (analysis, synthesis, evaluation and communication) to evaluate the current state of the platform, its limitations and possibilities. The literature is reviewed following this classification framework and it has been demonstrated the general lack of tailored built ICT tools to support ideas generation in particular in the area of AR and SAR technologies. The hardware and software components of the platform's setup has been described at the current state, highlighting which are the technological solutions selected and the functionalities provided to the users. As we tested the first version of SPARK with design practitioners in a controlled environment, we defined the design scenarios requirements for the SAR technology. The result is an overview on different SAR setups which demonstrate how the technological components of the platform should be configured according to specific design scenario and activity requirements. Nevertheless, in the SAR environment, the projection quality, the tracking of the blank mock-up and the interaction of the user with the mixed prototype have still some limitations that need to be further addressed. Future development steps, which are part of next development and testing activities, has been provided addressing the main challenges emerged. In particular, we provided solutions that could improve the engagement of the user in a shared design session and new advanced functionalities that could extend the creative capabilities of the codesign tool.

\section{Acknowledgment}

The work reported in this paper is part of the SPARK project, funded by the European Union's Horizon 2020 research and innovation programme under grant agreement No. 688417. This paper reflects only the authors' views and the European Commission is not responsible for any use that may be made of the information it contains. The authors would like to thank all the partners of the SPARK Consortium for the extensive support and valuable contribution provided in the development of this activity.

\section{References}

Ahlers, K.H., Kramer, A., Breen, D.E., Chevalier, P.-Y., Crampton, C. et al. (1995), "Distributed Augmented Reality for Collaborative Design Applications", Computer Graphics Forum, Vol. 14 No. 3, pp. 3-14. https://doi.org/10.1111/j.1467-8659.1995.cgf143_0003.x 
Becattini, N., Masclet, C., Ben-Guefrache, F., Prudhomme, G., Cascini, G. and Dekoninck, E. (2017), "Characterisation of a co-creative design session through the analysis of multi-modal interactions", Proceedings of ICED'17 / the 21 It International Conference on Engineering Design, Vol. 8, Vancouver, Canada, August 21-25, 2017, The Design Society, Glasgow, pp. 479-488.

Belcher, D. and Johnson, B. (2008), "MxR A physical model-based mixed reality interface for design collaboration, simulation, visualization and form generation", Proceedings of ACADIA $08 /$ the $28^{\text {th }}$ Annual Conference of the Association for Computer Aided Design in Architecture, Minneapolis, Minnesota, pp. 464471.

Ben Rajeb, S. and Leclercq, P. (2013), "Using Spatial Augmented Reality in Synchronous Collaborative Design", Proceedings of CDVE 2013 / the $10^{\text {th }}$ International Conference on Cooperative Design, Visualization, and Engineering, Mallorca, Spain, September 22-25, 2013, Springer, Berlin, Heidelberg, pp. 1-10. https://doi.org/10.1007/978-3-642-40840-3_1

Bimber, O. and Raskar, R. (2005), Spatial Augmented Reality: Merging Real and Virtual Worlds, A K Peters Ltd., Wellesley, Massachusetts.

Broll, W., Meier, E. and Schardt, T. (2000), "The virtual round table - a collaborative augmented multi-user environment", Proceedings of CVE'00 / the Third International Conference on Collaborative Virtual Environments, ACM Press, New York, USA, pp. 39-45. https://doi.org/10.1145/351006.351011

Calixte, X. and Leclercq, P. (2017), "The Interactive Projection Mapping as a Spatial Augmented Reality to Help Collaborative Design: Case Study in Architectural Design", Proceedings of CDVE $2017 /$ the $14^{\text {th }}$ International Conference on Cooperative Design, Visualization, and Engineering, Mallorca, Spain, September 17-20, 2017, Springer, Cham, pp. 143-152. https://doi.org/10.1007/978-3-319-66805-5_18

Chryssolouris, G., Mavrikios, D., Pappas, M., Xanthakis, E. and Smparounis, K. (2009), “A Web and Virtual Reality-based Platform for Collaborative Product Review and Customisation", In: Wang, L. and Nee, A.Y.C. (Eds.), Collaborative Design and Planning for Digital Manufacturing, Springer London, pp. 137-152. https://doi.org/10.1007/978-1-84882-287-0 6

Cross, N. (2008), Engineering Design Methods: Strategies for Product Design, 4th ed., J. Wiley and Sons Ltd.

Gervais, R., Roo, J.S. and Hachet, M. (2016), "Tangible Viewports", Proceedings of the TEI'16 / the 10th International Conference on Tangible, Embedded, and Embodied Interaction, Eindhoven, Netherlands, ACM Press, New York, New York, USA, pp. 176-184. https://doi.org/10.1145/2839462.2839468

Jones, J.C. (1984), “A method of Systematic Design”, In: Cross, N. (Ed.), Developments in Design Methodology, John Wiley \& Sons, Chichester, pp. 9-33.

Kiyokawa, K., Takemura, H. and Yokoya, N. (1999), "SeamlessDesign: a face-to-face collaborative virtual/augmented environment for rapid prototyping of geometrically constrained 3-D objects", Proceedings IEEE International Conference on Multimedia Computing and Systems, Vol. 2, Florence, Italy, June 7-11, 1999, IEEE, pp. 447-453. https://doi.org/10.1109/MMCS.1999.778493

Maher, M.L., Paulini, M. and Murty, P. (2011), "Scaling Up: From Individual Design to Collaborative Design to Collective Design", Proceedings of DCC'10 / the $4^{\text {th }}$ International Conference on Design Computing and Cognition, Springer, Dordrecht, pp. 581-599. https://doi.org/10.1007/978-94-007-0510-4_31

Nee, A.Y.C., Ong, S.K., Chryssolouris, G. and Mourtzis, D. (2012), “Augmented reality applications in design and manufacturing”, CIRP Annals, Vol. 61 No. 2, pp. 657-679. https://doi.org/10.1016/j.cirp.2012.05.010

Park, J. (2008), “Augmented Reality Based Re-formable Mock-Up for Design Evaluation”, Proceedings of 2008 International Symposium on Ubiquitous Virtual Reality, Gwangju, South Korea, July 10-13, 2008, IEEE, pp. 17-20. https://doi.org/10.1109/ISUVR.2008.22

Pei, E., Campbell, I. and Evans, M.A. (2009), "Building a Common Ground - The Use of Design Representation Cards for Enhancing Collaboration between Industrial Designers and Engineering Designers", Proceedings of the Design Research Society Conference 2008, Sheffield, UK, July 16-19, 2008, Sheffield Hallam University.

Seichter, H. (2003), “Augmented Reality Aided Design”, International Journal of Architectural Computing, Vol. 1 No. 4, pp. 449-460. https://doi.org/10.1260/147807703773633464

Shen, Y., Ong, S.K. and Nee, A.Y.C. (2010), "Augmented reality for collaborative product design and development", Design Studies, Elsevier, Vol. 31 No. 2, pp. 118-145. https://doi.org/10.1016/j.destud.2009.11.001

Wang, X. and Chen, R. (2009), "An experimental study on collaborative effectiveness of augmented reality potentials in urban design”, CoDesign, Vol. 5 No. 4, pp. 229-244. https://doi.org/10.1080/15710880903320020

Federico Morosi, PhD student in mechanical engineering

Politecnico di Milano, Mechanical engineering

Via Monte Santo 49, 21017 Samarate, Italy

Email: federico.morosi@polimi.it 